\title{
Pseudobasophilia as a Screening Tool in Dengue: A Single Center Study
}

\author{
Kevin Manuel ${ }^{1, \odot}$ Marie Moses Ambroise ${ }^{1} \quad$ Anita Ramdas ${ }^{1}$ Renu G'Boy Varghese ${ }^{1}$ \\ 1Department of Pathology, Pondicherry Institute of Medical \\ Address for correspondence Kevin Manuel, MD, Department of \\ Sciences, Kalapet, Puducherry, India \\ Pathology, Pondicherry Institute of Medical Sciences, Kalapet, \\ Puducherry 605014, India (e-mail: drkevinmanuel@gmail.com).
}

J Lab Physicians 2021;13:156-161.

\begin{abstract}
Objectives Proper serological testing for the definite diagnosis of dengue is costly and may not be easily available in a resource-limited setting. Hematological parameters can help in the early identification of dengue cases. This study aims to evaluate the occurrence and utility of pseudobasophilia in identifying dengue-affected patients.

Materials and Methods This retrospective cross-sectional study included 1,304 dengue cases confirmed by serology and 1,044 dengue serology negative acute febrile illness cases as controls. Complete blood count (CBC) values of the first EDTA (ethylenediamine tetraacetic acid) blood sample from automated hematology analyzers were reviewed. The hematological parameters in the dengue and control groups were compared and the variation of these parameters with the day of fever was also analyzed.

Statistical Analysis Mann-Whitney's test, Kruskal-Wallis test, and Fisher's exact test were used for statistical analysis. A $p$-value $<0.05$ was considered statistically significant for all tests.

Results There was a statistically significant variation between dengue cases and controls for hematocrit, platelet count, mean platelet volume, total white blood cell count, and absolute basophil count. The dengue group had a higher hematocrit from day 2 to day 10 , platelet count $\leq 100,000 / \mu \mathrm{L}$ from day 4 to day 9 , higher mean platelet volume from day 2 to day 7 , leucopenia from day 3 to day 5 , and higher absolute

Keywords

- dengue

- fever

- automated hematology

- lymphocytes basophil count from day 2 to day 10. Interestingly, pseudobasophilia was seen in 533 $(40.87 \%)$ of dengue cases and only $3(0.28 \%)$ of the controls. Pseudobasophilia was also observed to have an increasing trend to the day of fever.

Conclusion Pseudobasophilia along with other CBC parameters is useful and cost effective for the early identification of dengue. This can prompt early investigations and supportive treatment leading to improved clinical outcomes.
\end{abstract}

\section{Introduction}

Dengue is one of the most common causes of acute undifferentiated fever affecting both the pediatric and adult populations in India. ${ }^{1}$ It is a fast-spreading vector-borne disease that has a huge impact on public health. The disease is caused by the dengue virus, which is a small single-stranded RNA virus, transmitted to humans through the bite of the infected published online June 19, 2021
DOI https://doi.org/

$10.1055 / \mathrm{s}-0041-1730849$

ISSN 0974-2727 (c) 2021. The Indian Association of Laboratory Physicians.

This is an open access article published by Thieme under the terms of the Creative Commons Attribution-NonDerivative-NonCommercial-License, permitting copying and reproduction so long as the original work is given appropriate credit. Contents may not be used for commercial purposes, or adapted, remixed, transformed or built upon. (https://creativecommons.org/licenses/by-nc-nd/4.0/).

Thieme Medical and Scientific Publishers Pvt. Ltd. A-12, 2nd Floor, Sector 2, Noida-201301 UP, India 
female Aedes mosquito. The clinical symptoms include fever, headache, joint pain, and skin rashes. Following the onset of illness, thrombocytopenia is usually observed in the period between day 3 and day 8 . Unless detected early, the disease is fatal. ${ }^{2}$ In countries like India, being a dengue-endemic region, outbreaks have been associated with case fatality rates of 3 to $5 \%$. Most of the hospital centers in India are resource-limited and do not have proper dengue serological testing facilities, leading to poorer outcomes. ${ }^{3}$

Basophilia is defined as an absolute increase in the number of basophils. It is usually seen in myeloproliferative neoplasms such as chronic myeloid leukemia and also in allergic and inflammatory conditions. ${ }^{4}$ Hematology analyzers count basophils by electrical impedance and flow cytometric methods. Studies have shown that there is a poor correlation of basophil count between various instruments. ${ }^{5-7}$ Therefore, a proper peripheral smear examination is necessary for validation.

Pseudobasophilia is a cell counter analyzer phenomenon that has been caused by a cell population other than that of basophils. Some of the causes include leukemia, lymphoma, myeloma cells, and infectious mononucleosis. ${ }^{7.8}$ In a dengue-endemic country like India, pseudobasophilia is a common finding due to the presence of atypical lymphocytes. ${ }^{9,10}$ In the peripheral blood of patients, these machines tend to count atypical lymphocytes (or reactive lymphocytes) as basophils due to increased resistance to lysis. ${ }^{6}$

We can develop a simple and cost-effective technique in the laboratory using automated cell counter data to detect possible dengue cases and correlate this with the dengue NS1 antigen and immunoglobulin M (IgM) positivity. To our knowledge, this is the largest single center study to evaluate the relationship between dengue-affected population and pseudobasophilia. This study will also assess its consistency in identifying probable dengue cases and support in the preliminary assessment of acute febrile illness cases. This could lead to an overall improved clinical outcome through timely intervention and supportive therapy.

\section{Methods}

\section{Study Design and Data Collection}

This retrospective cross-sectional study was conducted at the Pondicherry Institute of Medical Sciences, Puducherry. We included all pediatric and adult age group patients who presented with acute febrile illness of less than 2 weeks duration between October 1 $1^{\text {st }}, 2016$ and November $1^{\text {st }}$ 2017. Clinical data were obtained from medical records. Complete blood count $(\mathrm{CBC})$, differential count, and peripheral smear data were recorded from the automated hematology analyzers and the medical records. All patients included in this study had a peripheral smear reported by a pathologist. Results of other relevant investigations like blood culture, scrub typhus IgM ELISA (enzyme-linked immunosorbent assay), widal test, leptospira IgM, chikungunya IgM, urine culture, malarial antigen test, sputum culture, GeneXpert Mycobacterium tuberculosis/rifampicin, stool for ova/cyst and culture, body fluids (pleural/ascitic/cerebrospinal fluid) culture, bronchoalveolar lavage culture, endotracheal aspirate culture, swab (throat/wound/vaginal/nasal) culture, HIV/hepatitis B virus/hepatitis C virus status, hepatitis A IgM, hepatitis E IgM, and Brucella agglutination test were also obtained retrospectively from the medical records and the Hospital Information Software (HIS). Automated hematology analyzers Horiba Pentra DF Nexus/DX Nexus (Horiba Medical, Montpellier, France) were used for CBCs in the study period.

Dengue patients were identified by a positive serology test for NS1 antigen and/or IgM antibody. Those patients who presented with fever due to various causes but were dengue serology negative during the same period were taken as the control group and these included bacterial infections (scrub typhus, enteric fever, primary bacteremia confirmed with blood culture, tuberculosis, and other respiratory illness), malaria, viral infections (H1N1, viral hepatitis, viral respiratory tract infections), and unknown causes of febrile illness. Cell counter data from the first EDTA blood sample of these patients were obtained from the automated hematology analyzers. The CBC data of follow-up samples sent on subsequent days was not collected. The data included hemoglobin, hematocrit, platelet count, mean platelet volume (MPV), white blood cell (WBC) count, differential count, absolute basophil count $(\mathrm{ABC})$, absolute neutrophil count, absolute lymphocyte count, absolute eosinophil count, and absolute monocyte count. We also obtained information regarding the day of fever when the first sample was collected.

\section{Exclusion Criteria}

We excluded the patients who developed fever after admission. Fever with localized causes, dengue fever cases with evidence of co-infection, and those with the unavailability of cell counter data were also excluded from the study.

After applying exclusion criteria, 1,304 dengue cases confirmed by NS1 antigen and/or IgM antibody and 1,044 dengue serology negative acute febrile illness cases (controls) were included in the study.

\section{Statistical Analysis}

The clinical and laboratory data for each patient were entered into a Microsoft Excel file. The mean, median, and standard deviation (SD) were calculated for continuous variables. Mann-Whitney's test, Kruskal-Wallis test, and Fisher's exact test were used for statistical analysis. A $p$-value $<0.05$ was considered statistically significant for all tests. Statistical analysis was performed using IBM SPSS Statistics for Windows, version 20.0 (IBM Corp., Armonk, New York, United States).

\section{Ethical Considerations}

This retrospective study was conducted after obtaining approval from the Institute Ethics committee (IEC: RC/18/53). Waiver of informed consent was granted for the study.

\section{Results}

In this study, we have included 1,304 dengue cases and 1,044 acute febrile illness cases (controls). The dengue group cases were solely positive for NS1 antigen in 787 
(60.3\%), dengue IgM antibody in 125 (9.6\%), and both in 392 (30.1\%) cases. Thirty-four cases (2.6\%) developed severe dengue. The control group consists of 344 cases of other viral illness, 419 cases of bacterial infections, 44 cases of malaria, 30 miscellaneous cases, and 207 cases of acute febrile illness where the exact etiology was not clear (undifferentiated fever).

Age and sex characteristics of dengue and the control group are shown in $\boldsymbol{- T a b l e} \mathbf{1}$. There was significant variation between dengue cases and controls for hematocrit, platelet count, MPV, total WBC count, and ABC as seen in - Table 2 . The $p$-value was $<0.001$ for hematocrit, platelet count, MPV, total WBC count, and ABC.

It was also interesting to note that hematological changes in the dengue group varied according to the day of fever. The data for the first ten days are shown in - Table 3 and - Fig. 1 .

Pseudobasophilia ( $\geq 2 \%$ ) was evident in 533 (40.87\%) of dengue cases and only three $(0.28 \%)$ of controls. Fisher's exact test showed this difference to be extremely significant ( $p$-value $<0.001)$. Hence it can be a good parameter for the early identification of dengue. - Table 3 also shows the relative frequency of pseudobasophilia with the day of fever in dengue cases. Pseudobasophilia was also evident in $22.1 \%$ of CBC samples on day 2 of fever, $29 \%$ on day 3, 39.2\% on day 4 , $48.2 \%$ on day $5,61.6 \%$ on day $6,58.3 \%$ on day 7 , and $61.5 \%$ on day 8 following which there was a decreasing trend from day 9 onwards. This is similar to $A B C$ which showed a significant variation with the day of fever.

Moreover, there are other characteristic hematological changes in dengue compared with other acute febrile illness cases as shown in $\boldsymbol{- T a b l e ~} \mathbf{2}$. These parameters also show a variation concerning the day of fever. Hence, pseudobasophilia with other characteristic hematological changes can be very useful to predict dengue.

Table 1 Baseline characteristics of dengue and control groups

\begin{tabular}{|l|l|l|}
\hline & Dengue group & Control group \\
\hline Mean age (years) & $28.62 \pm 15.6$ & $34.74 \pm 21.2$ \\
\hline Male & $808(62 \%)$ & $579(55.5 \%)$ \\
\hline Female & $496(38 \%)$ & $465(45.5 \%)$ \\
\hline
\end{tabular}

\section{Discussion}

In the dengue group, serology was solely positive for NS1 antigen in 787 (60.3\%), dengue IgM antibody in 125 (9.6\%), and both in $392(30.1 \%)$ cases. In comparison with a study done in Thailand, positive NS1 antigen was seen in $57.79 \%$ (89/154), dengue IgM antibody in $27.92 \%$ (43/154), and both in $14.29 \%(22 / 154) .^{11}$

We were also able to identify various hematological parameters that were significantly different between dengue and other acute febrile illnesses. These include hematocrit, platelet count, MPV, total WBC count, and ABC (- Table 2).

In comparison with the control group, the dengue group showed a higher hematocrit from day 2 to day 10 (highest on day 6). This was similar to the study from Thailand which also showed a higher hematocrit from day 3 to day 10 with the highest being on day $7 .{ }^{11} \mathrm{~A}$ higher hematocrit level in the dengue group is due to plasma leakage caused by increased vascular permeability. An in vitro study by Martina et al, showed that plasma leakage is due to apoptosis of endothelial cells which has been caused by the cross-reaction of proinflammatory cytokines and anti-NS1 antibodies with the surface proteins on these endothelial cells. ${ }^{12}$

Platelet count $\leq 100,000 / \mu \mathrm{L}$ was observed from day 4 to day 9 in our study (lowest on day 5) while in the Thailand study, it was from day 5 to day 8 (lowest on day 6 ). ${ }^{11}$ When compared with the control group, higher MPV was seen in the dengue group from day 2 to day 7 (highest on day 6). A study by Vogt et al determined that dengue virus infects human megakaryocytes in the in vitro, ex vivo, and in vivo models of infection. ${ }^{13}$ In addition, the function of platelets is disrupted due to the immunopathogenesis of dengue. The infection also induces platelet consumption and destruction due to disseminated intravascular coagulation, antiplatelet antibody activity, increased apoptosis, and activation of the complement system. ${ }^{14}$

In dengue, leucopenia is caused due to the ability of the virus to infect the bone marrow cells subsequently resulting in transient marrow suppression..$^{15}$ In our study, leucopenia was evident from day 3 to day 5 in comparison with the control group which was similar to the previously mentioned study. ${ }^{11} \mathrm{ABC}$ in the dengue group was higher from day 2 to day 10 (highest on day 8 ) of fever when compared with the control group.

Table 2 Comparison of CBC characteristics in dengue and control groups

\begin{tabular}{|c|c|c|c|c|c|c|}
\hline & & Hematocrit \% & Platelet count $\times 1,000 / \mu \mathrm{L}$ & MPV fL & Total WBC count $/ \mu \mathrm{L}$ & $\mathrm{ABC} / \mu \mathrm{L}$ \\
\hline \multirow[t]{3}{*}{ Dengue } & Mean & 39.2 & 92.6 & 9.1 & 4,800 & 75.2 \\
\hline & SD & 5.8 & 73.3 & 1.1 & 2,771 & 102.3 \\
\hline & Median & 38.9 & 75.0 & 9.1 & 4,100 & 43.1 \\
\hline \multirow[t]{3}{*}{ Controls } & Mean & 35.7 & 208.3 & 8.6 & 8,348 & 13.9 \\
\hline & SD & 6.5 & 103.2 & 0.8 & 5,044 & 22.3 \\
\hline & Median & 35.8 & 194.0 & 8.4 & 7,100 & 7.8 \\
\hline
\end{tabular}

Abbreviations: ABC, absolute basophil count; CBC, complete blood count; MPV, mean platelet volume; WBC, white blood cell. 


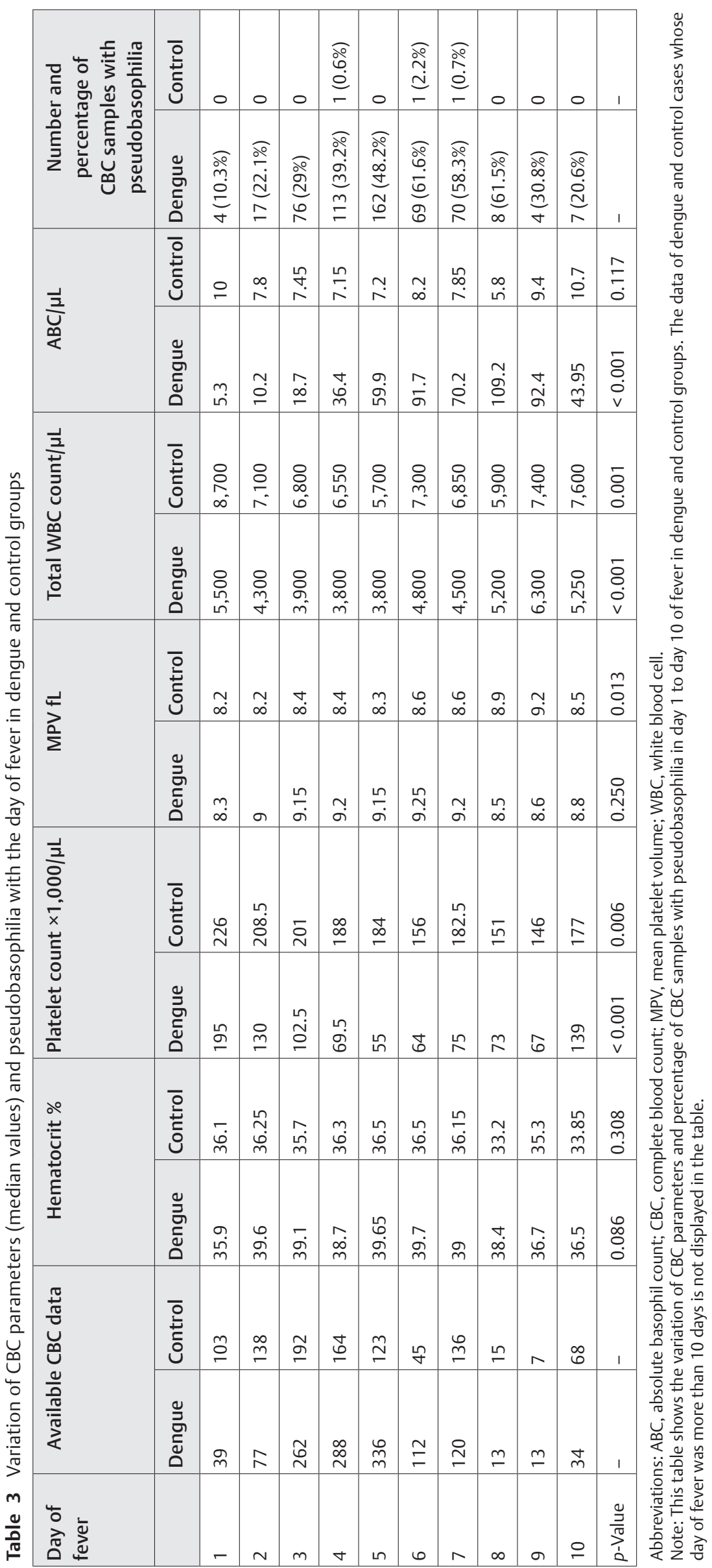




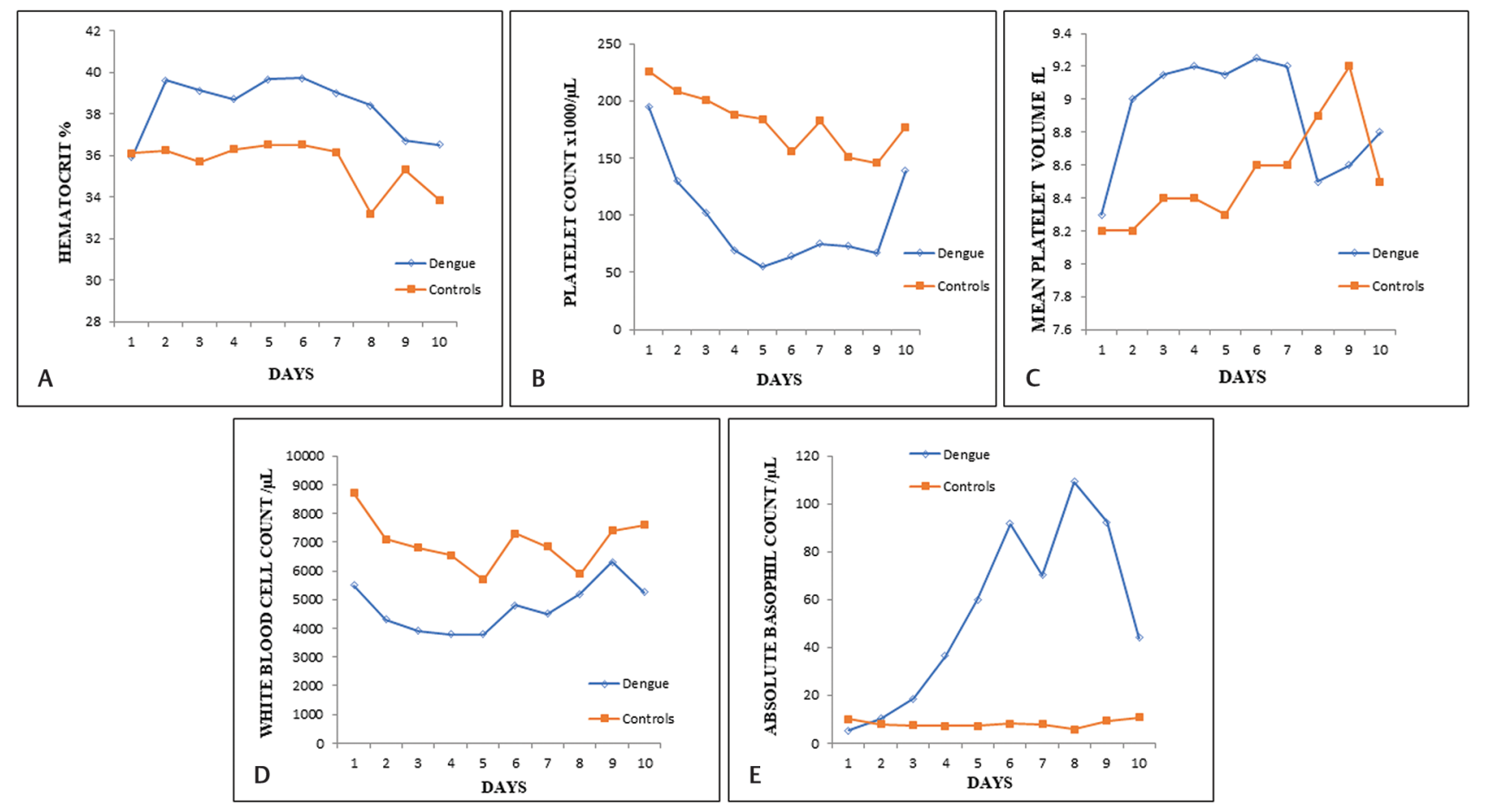

Fig. 1 Hematological variations in the dengue and control groups according to the day of fever. (A) Hematocrit variation. (B) Platelet count variation. (C) Mean platelet volume variation. (D) White blood cell count variation. (E) Absolute basophil count variation.

Pseudobasophilia ( $\geq 2 \%$ ) was observed in 533 (40.87\%) of dengue cases in our study. We noticed an increasing trend of pseudobasophilia in $\mathrm{CBC}$ samples from day 2 to day 8 of fever ( - Table 3). In another study from India, basophilia $>2 \%$ was seen in $52.9 \%$ of dengue patients..$^{10}$ In the Thailand study, basophil count was found to be not elevated..$^{11}$ In a study done in a different endemic area, dengue was responsible for $91.2 \%$ of cases with pseudobasophilia and thrombocytopenia on the Sysmex XE-2100. Peripheral smear examination of basophilia flags revealed reactive/atypical lymphocytes. ${ }^{9}$ This wide variation with the basophil counts could be due to the day of fever when the sample was collected, the duration of sample standing time, and the reagents used. Studies have also shown that there is a poor concordance between analyzers regarding the basophil count. ${ }^{6,8}$

Pseudobasophilia is an increase in basophil differential count as measured by the automated analyzer without an increase in the manual differential count. It is a cell counter analyzer phenomenon wherein cells with abnormal lymphocyte morphology/atypical cells in peripheral blood are falsely counted as basophils. Pseudobasophilia is known to occur in hematological malignancies. ${ }^{16}$ This phenomenon has been previously described in Technicon (Bayer Diagnostics, Tarrytown, New York, United States), ADVIA 120 (Siemens Medical Solutions Diagnostics, Tarrytown, New York, United States), and Sysmex XE-2100 (Sysmex Corporation, Kobe, Japan) automated cell counters. In the Sysmex XE-2100, atypical lymphocytes correlated with automated basophil counts. Pseudobasophilia phenomenon was observed more frequently in cases with "atypical lymphocytes" and "blasts" flags. ${ }^{17}$
This pseudobasophilia flag can be used to alert the pathologist/clinician to the presence of abnormal cells in the peripheral blood leading to early smear examination. ${ }^{8}$ Peripheral smear examination of all cases of pseudobasophilia in our study showed the presence of reactive/atypical lymphocytes. These reactive/atypical lymphocytes had a high nucleo-cytoplasmic ratio with deeply basophilic cytoplasm and homogenous/condensed nuclear chromatin with no nucleoli. True basophilia was not evident in any of the cases on peripheral smear examination. Pseudobasophilia in dengue is an underreported phenomenon. Our study does show a clear relationship between pseudobasophilia and dengue.

The most important merit of this study is the large sample size of dengue-affected individuals which has evaluated the relationship with pseudobasophilia. Since the cell counter CBC data of the first sample was collected at the time of admission, the treatment effects would be minimal.

The limitation of this study was that it was a retrospective study. There is a possibility of selection bias due to the collection of only dengue serology positive cases and false-negative cases could have been missed. The control group was made up of febrile patients with many diseases. Another limitation is that the serial monitoring of CBC data of each patient throughout their illness could not be done due to the retrospective nature of this study. A prospective study henceforth can correct these limitations.

\section{Conclusion}

From this study, pseudobasophilia can be used as an additional parameter in identifying probable dengue cases and 
also provide differentiation from other acute febrile illness cases. It can also be used with other CBC parameters to initiate early investigations and treatment leading to improved clinical outcomes, especially in a resource-limited setting.

\section{Authors' Contributions}

K.M. contributed toward the conception of idea, research design, data collection, statistical analysis, and report writing. M.M.A. did the research design, data collection, statistical analysis, report writing, and critical reviewing. A.R. and R.G.V. provided technical support and did report writing and critical reviewing.

\section{Institutional Review Board Approval}

This retrospective study was conducted after obtaining approval from the Institutional Ethics Committee [IEC: $\mathrm{RC} / 18 / 53]$. Waiver of informed consent was granted for the study.

\section{Conflicts of Interest}

No conflicts of interest.

\section{References}

1 Mørch K, Manoharan A, Chandy S, et al. Acute undifferentiated fever in India: a multicentre study of aetiology and diagnostic accuracy. BMC Infect Dis 2017;17(1):665

2 World Health Organization, Dengue: Guidelines for Diagnosis, Treatment, Prevention and Control: New Edition. Geneva: World Health Organization; 2009

3 Mutheneni SR, Morse AP, Caminade C, Upadhyayula SM. Dengue burden in India: recent trends and importance of climatic parameters. Emerg Microbes Infect 2017;6(8):e70

4 Galli SJ, Metcalfe DD, Arber DA, Dvorak AM, Basophils, mast cells, and related disorders. In: Kaushansky K, Lichtman MA, Prchal JT, et al, eds. Williams Hematology. 9th ed. New York: McGraw-Hill Education; 2016:971-972

5 Muniraj F, Amritraj V, Soundararajan G. Autoanalyzer generated spurious basophilia in adolescents and adults. Int J Sci Stud 2015;3(4):1-5
6 Amundsen EK, Henriksson CE, Holthe MR, Urdal P. Is the blood basophil count sufficiently precise, accurate, and specific?: Three automated hematology instruments and flow cytometry compared. Am J Clin Pathol 2012;137(1):86-92

7 Hur M, Lee YK, Lee KM, Kim HJ, Cho HI. Pseudobasophilia as an erroneous white blood cell differential count with a discrepancy between automated cell counters: report of two cases. Clin Lab Haematol 2004;26(4):287-290

8 Gibbs G, Campbell G, Christie I. Pseudobasophilia and the Advia 120. Hematology 2009;14(3):159-163

9 Pai S. Pseudobasophilia on the Sysmex-XE 2100: a useful screening tool for primary dengue infection in endemic area. Int J Lab Hematol 2012;34:25

10 Malathesha MK, Ashwini HN. Hematological manifestations in dengue fever-an observation study. J Evol Med Dent Sci 2014;3(9):2245-2250

11 Chaloemwong J, Tantiworawit A, Rattanathammethee T, et al. Useful clinical features and hematological parameters for the diagnosis of dengue infection in patients with acute febrile illness: a retrospective study. BMC Hematol 2018;18:20

12 Martina BE, Koraka P, Osterhaus AD. Dengue virus pathogenesis: an integrated view. Clin Microbiol Rev 2009;22(4):564-581

13 Vogt MB, Lahon A, Arya RP, Spencer Clinton JL, Rico-Hesse R. Dengue viruses infect human megakaryocytes, with probable clinical consequences. PLoS Negl Trop Dis 2019;13(11):e0007837

14 de Azeredo EL, Monteiro RQ de-Oliveira Pinto LM. Thrombocytopenia in Dengue: Interrelationship between virus and the imbalance between coagulation and fibrinolysis and inflammatory mediators. Mediators Inflamm 2015;2015:313842

15 Noisakran S, Onlamoon N, Hsiao HM, et al. Infection of bone marrow cells by dengue virus in vivo. Exp Hematol 2012;40(3):250-259.e4

16 Chandrashekar V. Basophil differentials as a marker for atypical lymphocyte morphologic characteristics. Lab Med 2013;44(2):133-135

17 Jácomo RH, Lozano VF, da Cunha Neto JG, Costa SS. What's the meaning of basophilia in Sysmex XE-2100? Arch Pathol Lab Med 2011;135(4):415 